\title{
Molecular Events in the Synthesis and Assembly of a Nicotinic Acetylcholine Receptor
}

\author{
D.J. ANDERSON* AND G. BLOBEL $\dagger$ \\ *Institute of Cancer Research, Columbia University College of Physicians and Surgeons, New York, New York 10032; \\ $\nmid$ Laboratory of Cell Biology, The Rockefeller University, New York, New York 10021
}

The acetylcholine receptor (AChR) has proven the most accessible model system for structure-function studies of a transmitter-activated ion channel (Karlin 1980; Conti-Tronconi and Raftery 1982). However, it also presents a good opportunity to study molecular events in the assembly of a multi-subunit integral membrane protein. Moreover, the synthesis of $\mathrm{AChR}$ is regulated during development, both by trophic factors (Jessel et al. 1979) and by activity (Brockes and Hall 1975). To understand the mechanistic bases of these regulatory influences, it would be helpful to know all the molecular events and intermediates in AChR biogenesis. We can presume that these events encompass gene transcription, mRNA processing and transport out of the nucleus, translation, subunit assembly, and intracellular transport of the subunits. The following discussion will concern what we have learned about the stages including and following mRNA translation.

To approach this problem we have used cell-free systems originally developed to study the transfer of secretory proteins across the rough endoplasmic reticulum (RER) membrane (Blobel and Dobberstein 1975). These systems consist of a standard mRNA in vitro translation system (from wheat germ or reticulocyte lysate), supplemented with canine pancreas rough microsomal membranes. These membranes are endowed with a set of specific proteins that can effect transfer of nascent secretory proteins across the lipid bilayer. First in the case of a viral spike glycoprotein, this system was also shown to reproduce accurately the synthesis, glycosylation, and asymmetric transmembrane insertion of a membrane protein. It has subsequently been successfully extended to eukaryotic membrane proteins, such as the H-2 antigen (Dobberstein et al. 1979), opsin (Goldman and Blobel 1981), and erythrocyte band III (Braell and Lodish 1982). Thus, this system is firmly established as a legitimate alternative to pulse-chase experiments in living cells as a means for studying membrane protein biogenesis. Moreover, it has the advantage that, because it constitutes a biosynthetic "dead end" (in the RER), certain intermediates can be trapped and studied in stable form in vitro, which would in living cells be transient, low-abundance species inaccessible for biochemical analysis.

To apply this approach, one needs a source of mRNA coding for the AChR subunits and antibodies with which to purify the translation products from the reaction mixtures by indirect immunoprecipitation
(Anderson and Blobel 1983b). In the case of the AChR, the electric organs of rays such as Torpedo have long been known to contain copious quantities of AChR protein and were therefore apparently the tissue of choice for extracting AChR mRNA. Unexpectedly, this tissue in fact contains very small amounts of mRNA (Anderson and Blobel 1983c). Apparently, the high steady-state levels of $\mathrm{AChR}$ in electric organ result from slow turnover of the protein. Although the tissue yields only small amounts of mRNA, this mRNA is enriched in AChR messages, each subunit's mRNA comprising about $0.5 \%$ of the total (Mendez et al. 1980).

Subunit-specific antibodies were obtained by isolating each subunit of the AChR from preparative SDS-polyacrylamide gels and injecting them into rats (Claudio and Raftery 1977; Lindstrom et al. 1978). As these antibodies had been raised against SDS-denatured material, we were able to demonstrate their subunit specificity using AChR in which the chains had been separated by SDS dissociation (Anderson and Blobel 1981). Furthermore, we could analyze the in vitro translation products by immunoprecipitation following SDS denaturation, a procedure that has proven helpful in analyzing the primary translation products of integral membrane proteins (Anderson and Blobel 1983b).

\section{Translation of AChR mRNA and Initial Membrane Insertion}

Analysis of the AChR subunits' primary translation products indicated that each polypeptide chain is translated from a separate mRNA. Thus, antibodies to each subunit immunoprecipitated a distinct translation product (Figs. 1 and 2). These products could each be labeled by formylated initiator methionyl-tRNA (Anderson and Blobel 1981), indicating that each subunit's mRNA has a distinct 5' AUG initiation codon. More recently, cloned cDNA probes have been used in Northern blot experiments to identify directly the separate mRNAs coding for each subunit (Noda et al. 1982, 1983; Sumikawa et al. 1982; Claudio et al. 1983; Devillers-Thiery et al. 1983). The synthesis of the AChR subunits can be contrasted with that of another multi-subunit receptor, the insulin receptor. In that case, the two subunits are synthesized as a polyprotein that is later cleaved into two distinct chains (Jacobs et al. 1983). These two alternative mechanisms for the synthesis of multi-subunit integral membrane proteins 


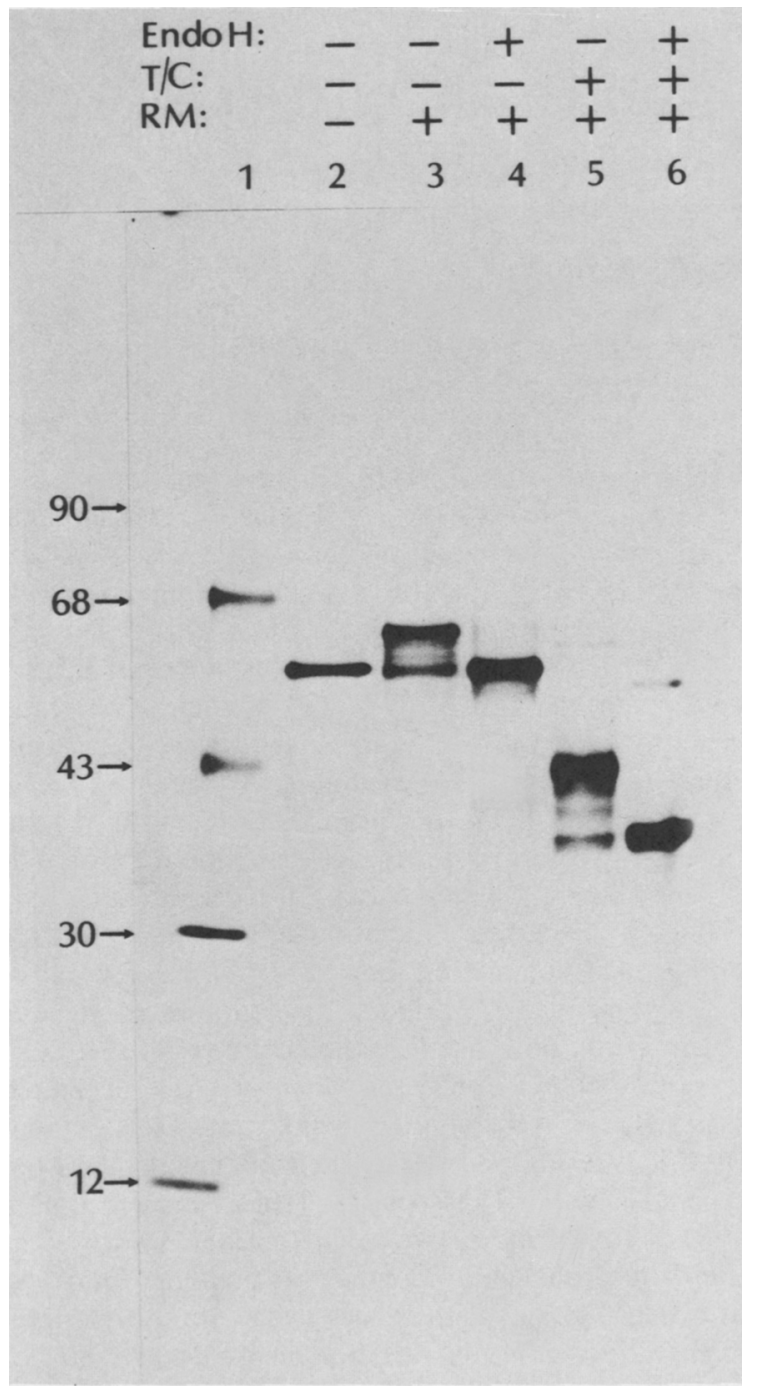

Figure 1. In vitro synthesis, glycosylation, and membrane insertion of AChR- $\delta$ subunit. Torpedo californica total cellular RNA was translated in a wheat germ cell-free system; AChR$\delta$ was purified by immunoprecipitation with anti- $\delta$ antibodies, and displayed by SDS-polyacrylamide gel electrophoresis and autoradiography. (Endo $\mathbf{H},+$ ) Immunoprecipitates were digested with Endo $\mathrm{H}$ before electrophoresis (Anderson and Blobel 1983c); (Endo H, -) samples were incubated in parallel without enzyme; (T/C) posttranslational incubation with trypsin and chymotrypsin; (RM) dog pancreas rough microsomes were included in the translation at $2 \mathrm{~A}_{280}$ units/ml final concentration. (Lane 1) ${ }^{14} \mathrm{C}$-labeled molecular weight markers. Note that the smallest protease-resistant fragment of AChR- $\delta$ in lane 5 comigrates with deglycosylated material in lane 6 and is therefore probably derived from a nonglycosylated, membrane-integrated form of $\mathrm{AChR}-\delta$.

have important consequences when considering the problem of quaternary assembly, as we shall discuss shortly.

The insertion of each subunit into the RER membrane occurs cotranslationally; that is, it is coupled to nascent polypeptide chain elongation. Insertion results in an asymmetric transmembrane orientation for each AChR subunit, an orientation in which part of each subunit is exposed to the cytoplasmic milieu and the remainder is either embedded in the membrane or exposed to the extracellular space. This has been demonstrated by so-called "protease-protection" experiments. Protease digestion of AChR subunits synthesized in the absence of microsomal membranes results in complete degradation of the chains, even if the chains are incubated with membranes after translation is complete (Anderson and Blobel 1981). In contrast, when microsomal membranes are included from the beginning of the translation incubation, posttranslational proteolysis results in only partial digestion of each AChR subunit (see, for example, Fig. 1, lane 5). This partial digestion is due to protection of most of the chain by the permeability barrier of the microsomal membrane, as evidenced by the fact that digestion is complete when the membranes are disrupted by detergent at the time of proteolysis. Thus, the heterologous cell-free system is able to reproduce the initial integration of the AChR subunits into the lipid bilayer of the RER. That this integration is correct is shown by the agreement between our data and those obtained for mature AChR in Torpedo plasma membranes (Strader and Raftery 1980; Wennogle and Changeux 1980; Froehner 1981; Wennogle et al. 1981).

\section{Glycosylation of the AChR Subunits}

Concomitant with their integration into the membrane, the four AChR subunits become core-glycosylated (Figs. 1 and 2). This glycosylation represents the first stage in a multistep process and consists of the attachment of preformed "core" oligosaccharide groups (Robbins et al. 1977) en bloc to Asn residues of the growing polypeptide chain (Rothman and Lodish 1977). Thus, core-glycosylation is a quantal event, and the AChR subunits differ in the number of these quanta that they receive. The $\delta$-subunit, for example, receives three core groups (Fig. 1, lane 3 ) that are susceptible to enzymatic removal by $\beta$ - $N$-acetylglucosaminidase $H$ (Endo H) (Fig. 1, lane 4), whereas the $\alpha$ - and $\beta$-subunits receive only one core group each (Fig. 2, lanes $R M+$ ). The number of these core oligosaccharide groups appears to be proportional to the increase in apparent molecular weight exhibited by the subunits when translated in the presence of microsomal membranes (compare Figs. 1 and 2, lanes RM,- , and RM, + Endo $H,-)$. These data are in good agreement with the cDNA-deduced amino acid sequences for these subunits, which reveal three Asn-X-Ser(Thr) sites in the $\delta$ subunit and one each in the $\alpha$ - and $\beta$-subunits (Noda et al. 1982, 1983). (The Asn-X-Ser[Thr] sequence constitutes the recognition site for the enzymes catalyzing core glycosylation.) The case of the $\gamma$-subunit is less clear-cut. The increase in the apparent $M_{\mathrm{r}}$ of the $\gamma$-subunit due to glycosylation appears commensurate with the attachment of three or four core oligosaccharides, by comparison to the $\delta$-, $\alpha-$, and $\beta$-subunits (Anderson and Blobel 1981). Indeed, the cDNA sequence of the $\gamma$ subunit shows four possible glycosylation sites (Noda 


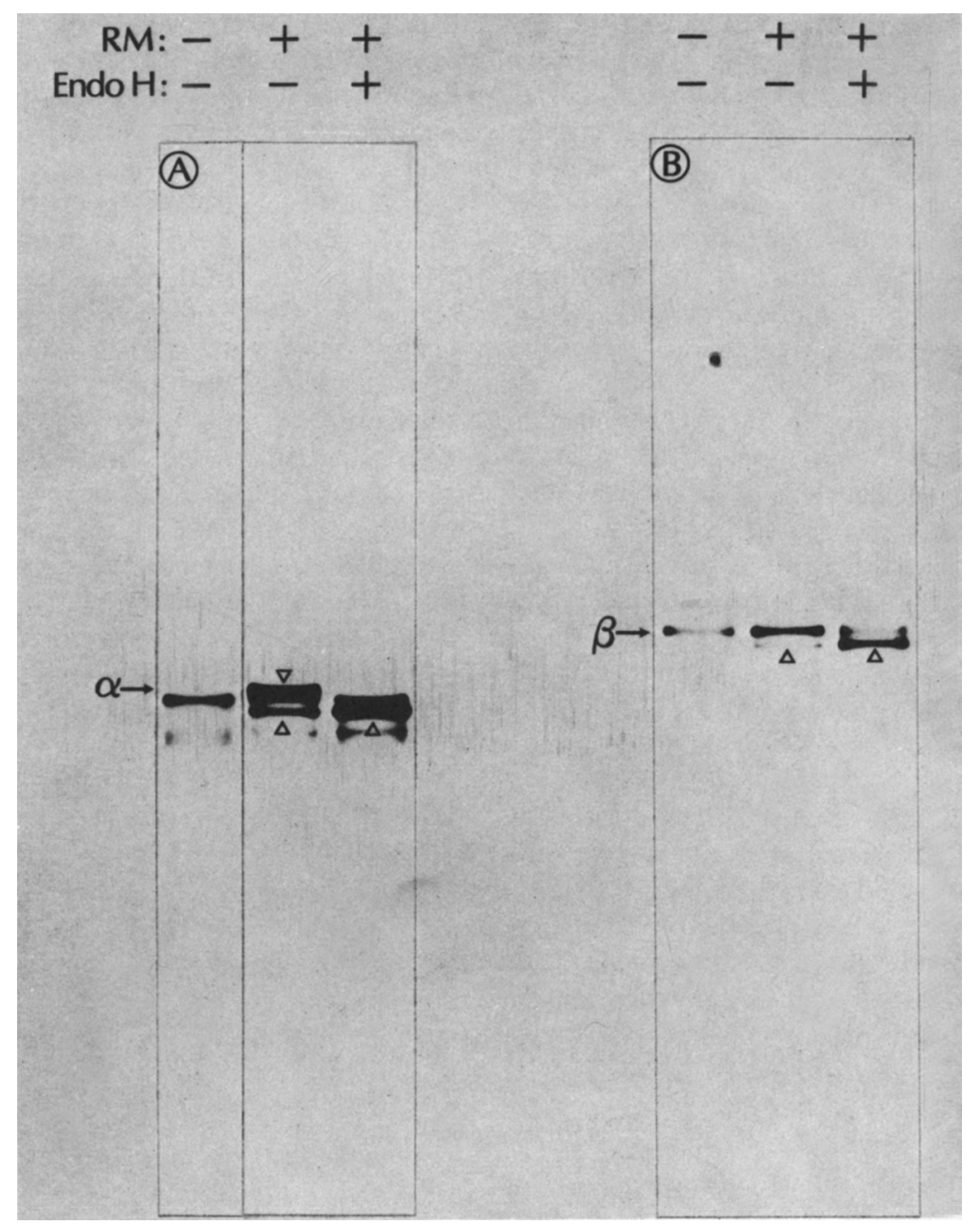

Figure 2. Glycosylation and signal peptidase cleavage of the $\alpha$ - and $\beta$-subunits. Immunoprecipitations were performed using either anti- $\alpha(A)$ or anti- $\beta(B)$ antisera. (RM) Rough microsomes were either included $(+)$ or omitted $(-)$ from the translation. Endo $\mathrm{H}$ incubations $(+$ or -$)$ were as per Fig. 1 . The position of the glycosylated form of each subunit is marked by a Greek character and horizontal arrow. Upward arrowheads indicate the signal peptidase-cleaved, but nonglycosylated form of each subunit. Note that the intensity of these bands increases after Endo $\mathrm{H}$ digestion.

et al. 1983). However, some secondary structure predictions for the $\gamma$-subunit place only two of these four sites extracellularly, where they could be accessible to the glycosyltransferases (Noda et al. 1983). It remains to be determined whether the error lies in the secondary structure model or in the inferred number of core sugar groups in the $\gamma$-subunit.

As expected (from the intracellular location of the core glycosylating enzymes), the carbohydrate residues of the subunits are located on the protease-resistant domain of each chain. Thus, the protease-resistant 44-kD fragment of the $\delta$-subunit (Fig. 1, lane 5) is Endo-Hsensitive (Fig. 1, lane 6). Interestingly, all four subunits' protease-resistant domains are converted to bands of about the same size (ca. $37 \mathrm{kD}$ ) by enzymatic removal of the carbohydrate (D.J. Anderson, unpubl.). As the intact subunits differ in their apparent $M_{\mathrm{r}}$, this observation of similar-sized membrane-protected domains suggests that the mass differences between the subunits can be accounted for by the relative sizes of their protease-susceptible cytoplasmic domains, as suggested by others (Conti-Tronconi and Raftery 1982). This finding is consistent with secondary structure predictions for the four subunits based on cDNA sequence analysis (Noda et al. 1983). It is further supported by the direct identification of different-sized cytoplasmic domains, released from some of the subunits by proteolysis and recovered by monoclonal antibody precipitation (Anderson et al. 1983).

\section{Role of the Signal Peptide \\ in Membrane Insertion of the AChR Subunits}

Many of the single-subunit membrane proteins studied previously are synthesized with short, aminoterminal peptide extensions termed signal sequences (Lingappa et al. 1978). These extensions are removed by a specific endoprotease shortly after insertion of the protein into the membrane (Blobel and Dobberstein 1975). They are shared by all proteins that undergo 
cotranslational transfer across the RER membrane, including secretory, lysosomal, and prokaryotic secretory proteins (Blobel 1980). Without exception, those membrane proteins whose signal peptide is proteolytically removed have their amino termini exposed on the outer surface of the membrane. There is also a class of membrane proteins that do not undergo proteolytic removal of a signal peptide (Mostov et al. 1981; Braell and Lodish 1982). Several proteins in this class have their amino termini exposed on the cytoplasmic side of the membrane (Braell and Lodish 1982).

To which class of proteins do the AChR subunits belong? We determined by direct radiosequence analysis that $\mathrm{AChR}-\delta$ contained a 21 -amino-acid-long signal peptide that was proteolytically removed following membrane insertion (Anderson et al. 1982). Consistent with this finding, the amino terminus of the $\delta$ subunit was found to be protected by the microsomal membrane from proteolysis, and thus is most likely extracellularly exposed (Wennogle et al. 1981; Anderson et al. 1982). Our partial sequence for the $\delta$ signal peptide was subsequently confirmed by the cDNA sequence (Noda et al. 1983), and cDNA clones revealed signal sequences for the other three subunits as well (Sumikawa et al. 1982; Noda et al. 1982; Claudio et al. 1983; Devillers-Thiery et al. 1983). The secondary structure models based on these cDNA sequences also predict an extracellular disposition for the subunits' amino termini, in agreement with our data for the $\delta$ subunit.

What role does the signal peptide play in the insertion of the AChR subunits into the RER membrane? Early on (Milstein et al. 1972; Blobel and Dobberstein 1975), it was proposed that signal sequences contain the information that "targets"' nascent proteins specifically to the RER. It was also suggested that the signal peptide (perhaps by virtue of its hydrophobicity) initiated transfer of the growing polypeptide across the membrane, once a functional ribosome-membrane junction had formed (Blobel 1980). Recently, an 11 S ribonucleoprotein complex has been purified that appears to decode the information contained in secretory protein signal peptides (Walter and Blobel 1980, 1982). Termed SRP (signal recognition particle), this complex consists of six protein subunits together with one molecule of $7 \mathrm{~S}$ RNA, and forms a high-affinity "bridge" between polysomes whose nascent proteins contain signal sequences, and an integral membrane protein receptor in the RER (Gilmore et al. 1982; Meyer et al. 1982). This arrangement somehow permits the initiation of translocation of the nascent chain across the lipid bilayer.

SRP is likewise required for the insertion of the AChR subunits into the RER membrane. This was expected based on the structural similarities between signal sequences from secretory and membrane proteins and from functional similarities suggested by competition experiments (Lingappa et al. 1978). Thus, rough microsomes (RM) from which SRP has been extracted by a $0.5 \mathrm{M}$ KOAc wash (K-RM) (Warren and Dobberstein 1978; Walter and Blobel 1980) are in- capable of integrating or glycosylating AChR- $\delta$, for example (Fig. 3, lane 3). When purified SRP is added back to the system at the beginning of translation, the integration and glycosylation of AChR- $\delta$ are restored (Fig. 3, lanes 4 and 5). Interestingly, in the absence of K-RM, SRP inhibited the synthesis of AChR- $\delta$ (Fig. 3, lane 2 ). In the case of a secretory protein, prolactin, this inhibition was shown to be due to a site-specific elongation arrest that occurs as soon as the signal sequence emerges from the ribosome (Walter and Blobel 1981), but which is released by the SRP receptor (Gilmore et al. 1982). We may assume that a similar arrest accounts for the observed inhibition of AChR- $\delta$ synthesis. Thus, the insertion of the AChR subunits into the membrane utilizes the same protein machinery that initiates the complete transfer of secretory proteins across the membrane.

In the case of membrane proteins, however, transfer of the nascent polypeptide across the membrane is interrupted, presumably due to the emergence of appro-

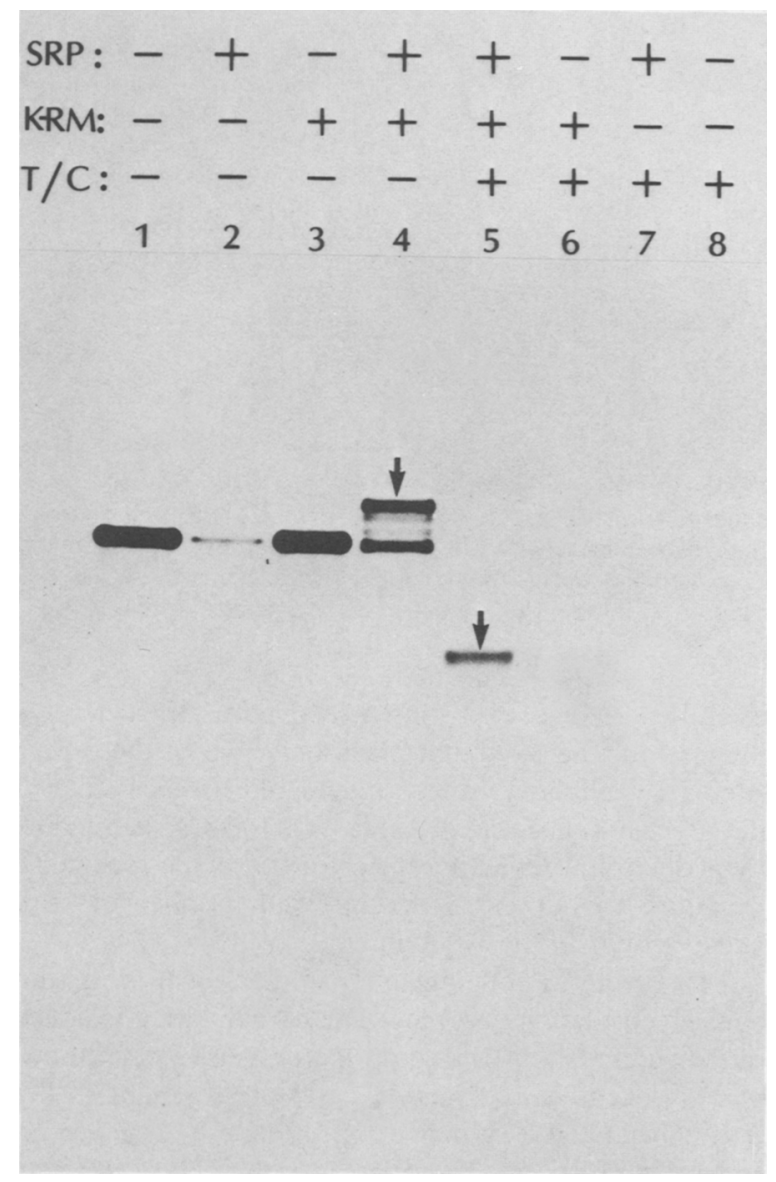

Figure 3. SRP-dependent glycosylation and membrane integration of AChR- $\delta$ subunit. (SRP) Signal recognition particle was included $(+)$ at $220 \mathrm{units} / \mathrm{ml}$ or omitted $(-)$ from the reaction; (K-RM) RM extracted with $0.5 \mathrm{~m} \mathrm{KOAc}$ were included $(+)$ or omitted $(-)$; (T/C) trypsin/chymotrypsin digestion as per Fig. 1. Arrows in lanes 4 and 5 indicate the fully glycosylated form of AChR- $\delta$ and its corresponding membrane-protected fragment, respectively. 
priate hydrophobic "membrane anchor" sequences or "stop-transfer" sequences from the ribosome (Blobel 1980; Boeke and Model 1982). The case of the AChR subunits is even more complex, in that secondary structure models predict four transmembrane segments for each subunit (Claudio et al. 1983; Devillers-Thiery et al. 1983; Noda et al. 1983). It is generally agreed upon by theorists that such multiple transmembrane segments are likely to be inserted into the membrane in pairs, as hairpin loops (Blobel 1980; Engleman and Steitz 1981). However, it is not yet known whether the insertion of such loops requires an internal signal sequence that is acted upon by the SRP-SRP receptor machinery. If the exclusive function of the signal sequence is that of targeting the protein to the correct intracellular membrane, then one per protein should be sufficient. The integration of subsequent helices into the membrane would then occur by insertion sequences (Blobel 1980), such as those that anchor the protein cytochrome $b_{5}$ in the membrane (Rogers and Strittmatter 1975) and that apparently partition spontaneously into the lipid bilayer by virtue of their hydrophobicity.

\section{Posttranslational Assembly of AChR}

The AChR subunits are not assembled with one another into a functional complex immediately upon insertion into the RER membrane. Rather, multimeric assembly of AChR appears to be a lengthy, posttranslational process. Thus, the in-vitro-synthesized and membrane-integrated $\alpha$-subunit does not exhibit high-affinity binding to $\alpha$-bungarotoxin (Anderson and Blobel 1981). Nor is any inter-subunit interaction observed in this system (see below). As it is currently not feasible to reconstruct the movement of proteins from the RER to the Golgi apparatus in vitro, we turned to a cellculture system to investigate the formation of the toxinbinding site. Initially, we identified the $40-\mathrm{kD}(\alpha)$ subunit of the AChR in cultured chick embryonic myotubes by a two-step affinity purification procedure involving first cobra toxin-Sepharose and then concanavalin A-Sepharose (Fig. 4, lanes 1 and 2). The 40-kD polypeptide enriched by this procedure was eliminated from the preparation by including competing cholinergic ligands in the toxin-Sepharose step (Fig. 4, lane 3), and was specifically immunoprecipitated by antisera to the Torpedo AChR (Fig. 4, lane 4).

We used this two-step procedure to purify AChR from cultures that were pulsed for 5 minutes with $\left[{ }^{35} \mathrm{~S}\right]$ methionine and then chased for various periods of time in the presence of cold methionine and puromycin (to inhibit further protein synthesis). In agreement with our in vitro data, no toxin-binding $40-\mathrm{kD}$ material could be recovered from cells immediately following the pulse (Fig. 5B, lane 1). With increasing times of chase, however, increasing amounts of the $40-\mathrm{kD}$ subunit bound to the toxin-Sepharose column (Fig. 5B, lanes 2-4), although no further protein synthesis occurred during this period (Fig. 5A, lanes 1-4). Thus, we may conclude from these data that the formation of

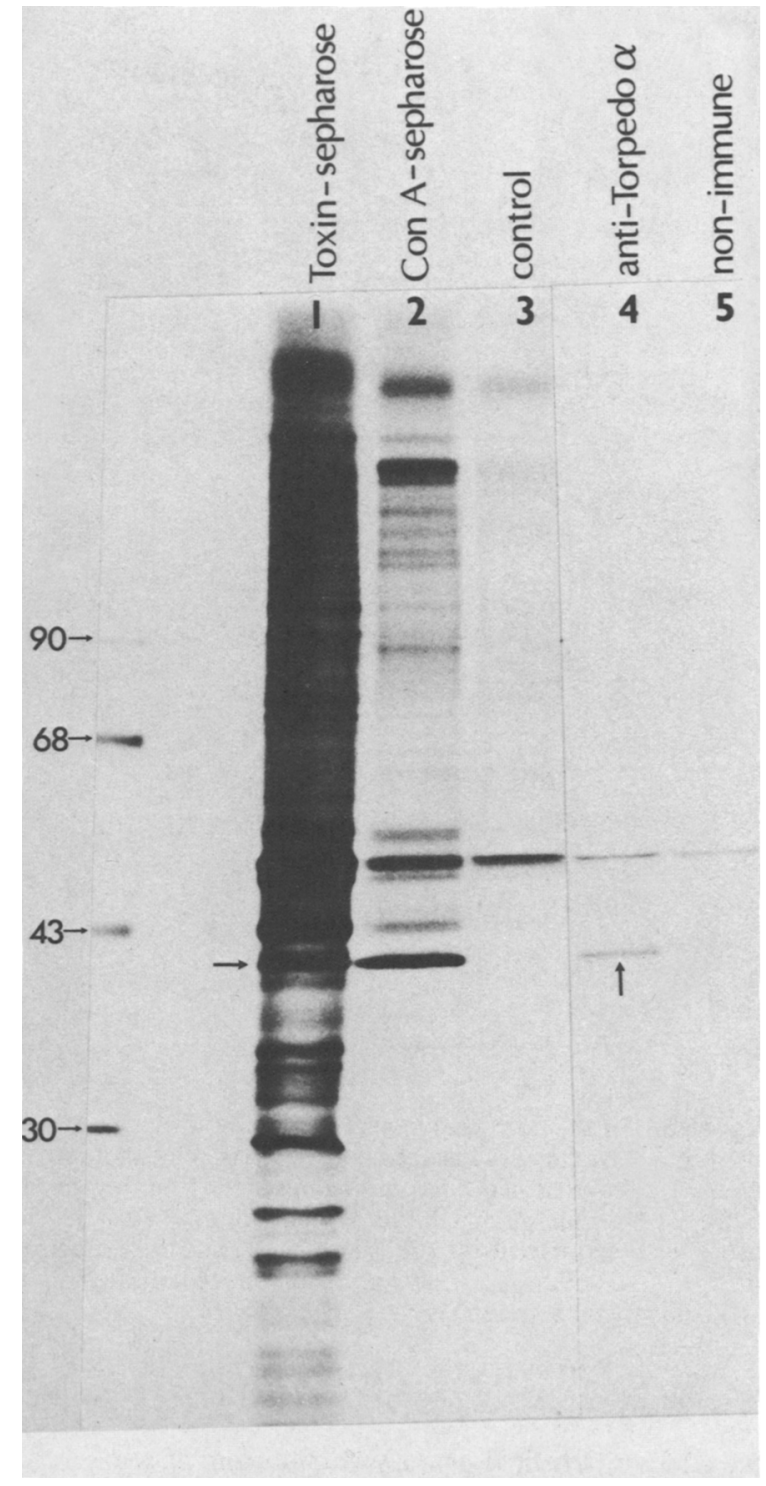

Figure 4. Identification and partial purification of an $M_{\mathrm{r}}$ 40,000 subunit of embryonic chick muscle AChR. (Lane 1 ) Material retained by the cobra toxin-Sepharose affinity resin. (Lane 2) An aliquot of the material in lane 1 was adsorbed to Con A-Sepharose, and bound material was eluted directly into SDS gel sample buffer. (Lane 3) As in lane 2 except that 125 nM free $\alpha$-bungarotoxin was present during the initial incubation with cobra toxin-Sepharose. (Lanes 4 and 5) Aliquots of the material in lane 2 were immunoprecipitated with antiTorpedo $\alpha$-subunit antiserum (lane 4 ) or nonimmune serum (lane 5).

the high-affinity toxin-binding site is a posttranslational event that occurs in the absence of ongoing protein synthesis, over a period of about 30 minutes. A similar observation has been made by Merlie and Sebbane (1981) using a tumor cell line $\mathrm{BC} 3 \mathrm{H}-1$.

We attempted to relate temporally the formation of the toxin binding-site to some carbohydrate modifications that occur in the Golgi apparatus. Specifically, the core oligosaccharides of some membrane proteins have been shown to become resistant to Endo $\mathbf{H}$ cleavage as 


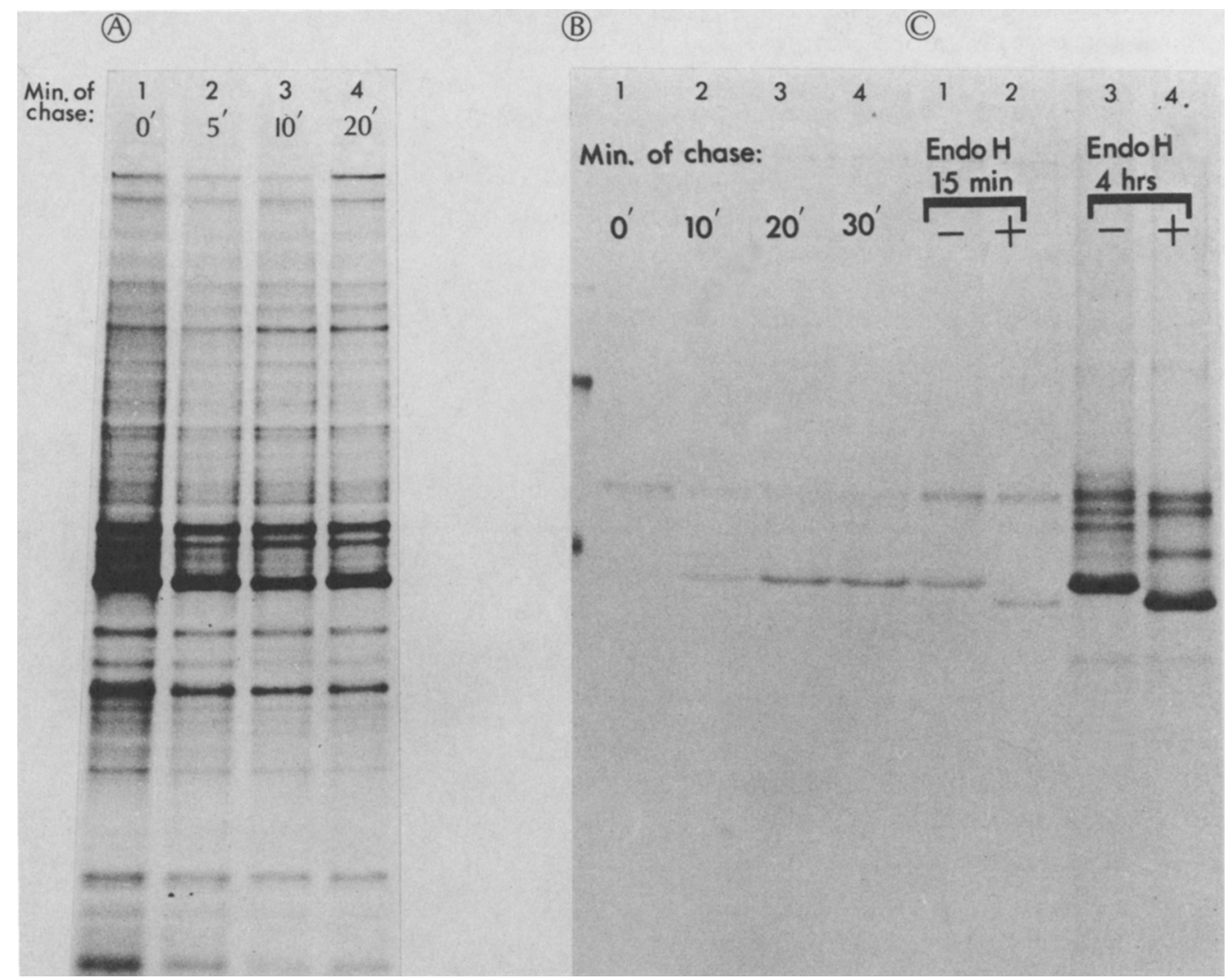

Figure 5. Pulse-chase kinetic analysis of the formation of the toxin-binding site in the $40-\mathrm{kD}$ polypeptide. $(A)$ Demonstration that protein synthesis is instantaneously stopped by addition of puromycin. Cells were pulsed for 5 min with [ ${ }^{35}$ S]methionine and chased for the indicated times in the presence of puromycin. Labeled proteins solubilized by $1 \%$ Triton X-100 were analyzed by SDS gel electrophoresis and fluorography for each time point. $(B)$ Cells were pulsed and chased as in $A$. For each time point, the dishes were extracted with $1 \%$ Triton X-100 and the extracts subjected to the toxin-Sepharose/Con A-Sepharose isolation scheme of Fig. 4. (C) Cells were labeled for the times indicated above the brackets, $\mathrm{AChR}$ isolated, and subjected to Endo $\mathrm{H}$ digestion $(+)$ prior to electrophoresis.

the result of terminal glycosylation during passage from the cis to trans compartments of the Golgi apparatus (Dunphy et al. 1981). Unfortunately, AChR- $\alpha$ showed no such changes in Endo $H$ sensitivity during its passage through the cell (Fig. 5C, lanes 2 and 4). Thus, it is not possible to say whether formation of the toxin-binding site in the $\alpha$-subunit precedes transport to the Golgi apparatus. As toxin-binding sites have been seen by electron microscope autoradiography over the Golgi region but not the RER or transitional elements (Fambrough and Devreotes 1978), it is possible that the toxin-binding site forms after transport to the Golgi.

Formation of the toxin-binding site does not require association of the $\alpha$-subunit with other subunits (Merlie et al., this volume). Indeed, $\alpha$-subunit purified by preparative SDS-polyacrylamide gel electrophoresis of the mature AChR complex can be reconstituted, after detergent removal, into a form that displays some (but not all) characteristics of high-affinity $\alpha$-bungarotoxin binding (Tzartos and Changeux 1983). This is to be contrasted with the $\alpha$-subunit in the RER, which apparently displays no such activity. The difference be-

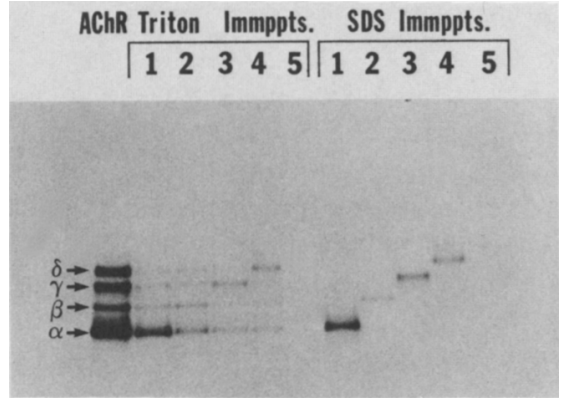

Figure 6. Characterization of anti-AChR subunit antisera under denaturing versus nondenaturing conditions. Triton $\mathrm{X}-100$ immunoprecipitates: Immunoprecipitation was performed after dilution of 9S, ${ }^{125}$-labeled $\mathrm{nAChR}$ into immunoprecipitation buffer containing $1 \%$ Triton $X-100$ and $0.2 \%$ SDS. SDS immunoprecipitates: Immunoprecipitation was performed after prior denaturation of the ${ }^{125}$ I-labeled AChR in $1 \%$ SDS. Antisera used in lanes $1-5$ were anti- $\alpha,-\beta,-\gamma,-\delta$, and nonimmune, respectively. The nonstoichiometric recovery of the four subunits in each of the Triton X-100 immunoprecipitates is most likely due to partial dissociation of the AChR complex arising from the iodination procedure (Anderson and Blobel 1981). 
tween the two is likely to be due to some as yet undetected posttranslational, covalent (i.e., SDS-resistant) modification that occurs after transport out of the RER. A possible candidate for this is $\boldsymbol{O}$-glycosylation of serine and threonine hydroxyl groups by hexoses and hexosamines, which is catalyzed by glycosyltransferases located in the Golgi apparatus (Hunt and Summers 1976).

\section{AChR Subunits Appear to Self-associate in the RER}

Quaternary assembly of the AChR subunits may take at least as long as acquisition of the toxin-binding site by the $\alpha$-subunit, if not longer (Merlie et al., this volume). When we immunoprecipitated in-vitro-synthesized and membrane-inserted AChR subunits after solubilization of the membrane with Triton X-100 (which does not dissociate the mature AChR complex), only one subunit was brought down by each subunitspecific antibody (not shown), even though all four subunits of mature, 9S AChR were coprecipitated by this procedure as expected (Fig. 6, Triton Immppts.). Surprisingly, however, when we analyzed these Tritonsolubilized in-vitro-synthesized AChR subunits by sucrose gradient centrifugation, we found that they migrated as greater than monomeric species (shown for the $\delta$-subunit in Fig. 7). Immunoprecipitation and electrophoretic analysis showed that these complexes were formed by each subunit, independent of the others, even though all four subunits were synthesized in the same reaction mixture (Anderson and Blobel 1981). These complexes, or homo-oligomers, were seen under a variety of conditions of salt, $\mathrm{pH}$, divalent cation, and type of detergent (Anderson and Blobel 1983a). Most important, they were not observed for another unrelated membrane protein, opsin, studied in exactly the same way (Fig. 8). As opsin shares the cotranslational integration process in common with the AChR subunits (Goldman and Blobel 1981), the high S values seen for the AChR subunits are unlikely to be due to an association with nonradioactive components of the RER translocatich machinery (such as SRP or SRP receptor), as opsin would in that case exhibit a similar sedimentation profile. However, we cannot rigorously rule out the possibility that the AChR subunits all aggregate nonspecifically with some unlabeled RER proteins, which do not interact with opsin.

Although it is not yet clear that the homo-oligomeric forms of the AChR subunits seen in vitro exist in vivo (cf. Merlie et al., this volume), these structures can be rationalized as potential metastable intermediates in quaternary assembly. Structural studies of AChR (Kistler et al. 1982; Karlin et al. 1983) suggest that the subunits surround a central ion channel like barrel staves. If each subunit formed a boundary of the ionophore, it would be expected to contain a surface rich in charged or polar amino acid side chains within the membrane (cf. Fairclough et al., this volume). As the subunits are each inserted separately into the membrane, assembly of the ion channel cannot occur co-
(A)

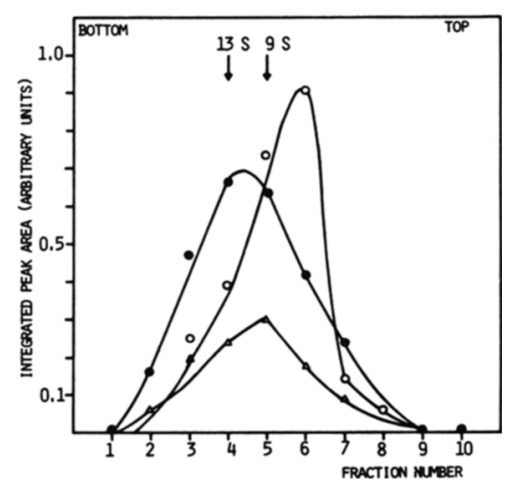

(B)

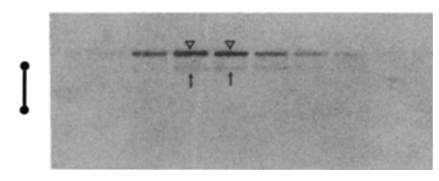

(C)

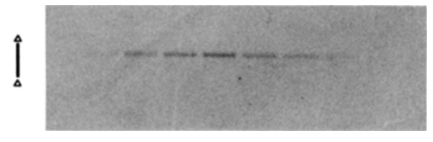

(D)

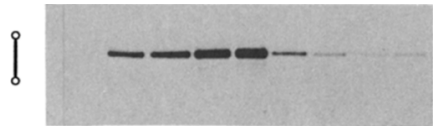

(E)

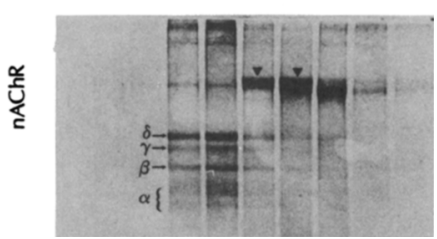

Figure 7. Sucrose gradient analysis of in-vitro-synthesized AChR- $\delta$. (A) Quantitative representation of the gel profiles shown in $B-D$, obtained by a densitometric scan of the autoradiographic films. The band quantitated by this procedure is indicated (in all ensuing figures as well) by an open arrowhead, wherever multiple forms appear on the gel. $(A[\theta]$ and $B$ ) AChR- $\delta$ obtained by analysis of total reaction mixture. Upward arrows in $B$ indicate the nonglycosylated and partially glycosylated forms characterized previously; open arrowheads indicate the fully glycosylated form (Fig. 1). ( $A$ $[\triangle]$ and $C$ ) AChR- $\delta$, solubilized from reisolated microsomes at a fourfold greater dilution than that used in $B$. $(A[O]$ and $D$ ) Pre-AChR- $\delta$, synthesized in the absence of microsomal membranes, analyzed on gradients not containing detergent. (E) AChR-enriched Torpedo membranes solubilized in $1 \%$ Triton X-100/0.3 M KOAc and analyzed in parallel with the samples in $B-D$. However, the gradient fractions were precipitated with trichloracetic acid for analysis of the total protein components; shown is the Coomassie blue-stained gel. The four AChR subunits are indicated by horizontal arrows; the $\alpha$-chain runs as a smeared doublet due to heating and trichloroacetic acid preparation. Arrowheads indicate the putative large subunit of the $\mathrm{Na}^{+}-\mathrm{K}^{+}$ATPase. Arrows in $A$ indicate the peak fractions ( 4 and 5 ) containing $\mathrm{nAChR}$ (see $E$ ). Fraction 4 is calculated to be $12 S$, whereas fraction 5 is 9S. Although we have assumed (arrows) that fraction 4 contains some $13 \mathrm{~S} \mathrm{AChR}$, the resolution of our fractionation is not sufficient for us to determine the actual amount of dimeric receptor. Each fraction spans ca. $3 S$ units. 


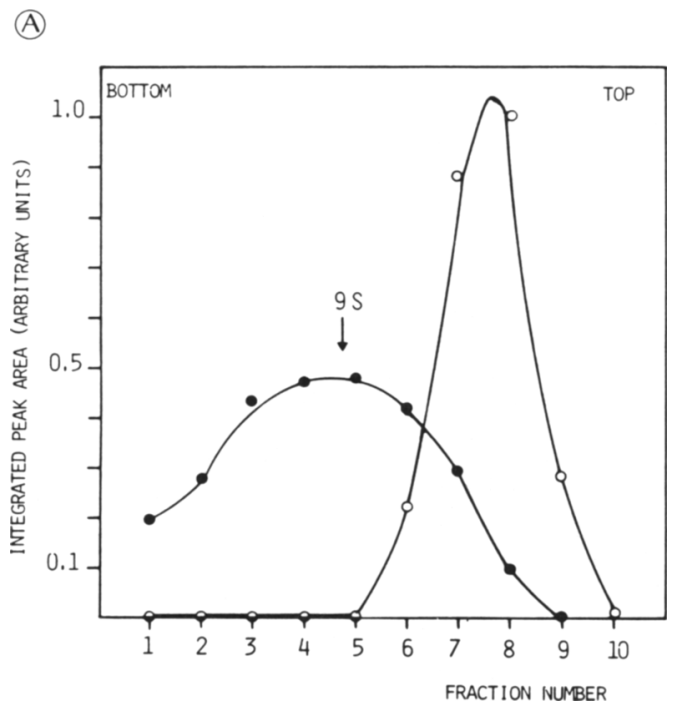

(B)

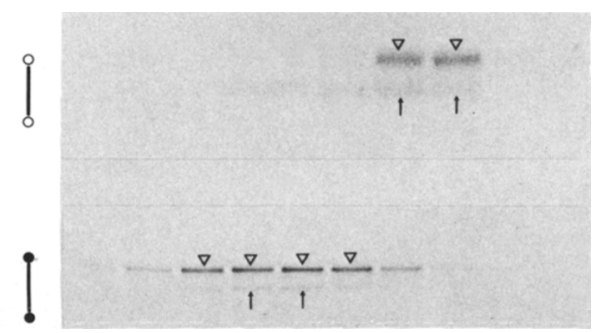

Figure 8. Comparative sedimentation analysis of $\mathrm{AChR}-\delta$ and opsin. $(A)$ Sedimentation profiles reconstructed from densitometric scans of the gels shown in $B$ and $C$. ( $A[O]$ and $B$ ) Opsin. Arrowheads indicate the core-glycosylated, membrane-integrated form of opsin (Goldman and Blobel 1981); upward arrows indicate the residual unintegrated precursor (or integrated, nonglycosylated material), which smears slightly into the gradient. ( $A[\theta]$ and $C$ ) AChR- $\delta$ analyzed in parallel. Arrowheads indicate the fully glycosylated form; upward arrows indicate the residual precursor. AChR- $\delta$ in this experiment assumed a' slightly broader distribution than on other occasions.

translationally and these ionophore boundary surfaces could in the interim be in direct contact with the interior of the lipid bilayer. However, it might be energetically preferred for the subunits to adopt a homo-oligomeric configuration, in which the hydrophilic ionophore surfaces were sequestered in the interior of aggregates formed by multiple copies of the same subunit. This configuration would maximize contact between the polar ionophore surfaces and minimize their interaction with the hydrophobic interior of the bilayer. These homologous associations could form either during or immediately after translation, when the local concentration of subunit in the membrane would be extremely high (due to the multiple copies of each chain being synthesized from the same mRNA). Irrespective of the thermodynamic rationale, homo-oligomers could provide a useful way of "packaging" the subunits for transport out of the RER to the Golgi apparatus. Finally, the homooligomer configuration is attractive for evolutionary reasons: As the four AChR subunits seem to have evolved by a process of gene duplication and divergence (Raftery et al. 1980; Noda et al. 1983), it is likely that the ancestral AChR may have itself been a homo-oligomer, as is the contemporary gap junction channel (Hertzberg and Gilula 1982). The assembly of the contemporary AChR could in this light be considered (tongue-in-cheek) as an example of "ontogeny recapitulates phylogeny" at the molecular level.

If the homo-oligomer precursor model is correct, subunit-subunit exchange must eventually occur in AChR assembly. These exchanges must result in heterologous subunit associations that are of higher affinity than the homologous ones, to be stable. This would occur if the homologous associations were particularly weak, or if the heterologous chain interactions were somehow rendered irreversible once they occurred. The broad sedimentation profile of the homo-oligomers (Figs. 7 and 8) is consistent with their being weakly associated. On the other hand, the heterologous associations could be rendered irreversible by a covalent modification. If they indeed occurred in the Golgi apparatus, for example, heterologous chain interactions might cause conformational changes exposing Ser or Thr hydroxyl groups to glycosyltransferases; glycosylation of these side chains could render this conformational change irreversible. The strong prediction of this model is that inhibition of $O$-glycosylation would block assembly of the AChR subunits. It has been shown that inhibition of $\mathrm{N}$-glycosylation by tunicamycin blocks maturation of the $\alpha$-bungarotoxin-binding site in the $\alpha$-subunit (Merlie et al. 1981); however, it is possible that the direct effect in this case was simply to prevent transport of AChR out of the RER to the Golgi apparatus. This result points up the current lack of a good cell culture system in which to study the actual assembly of all the AChR subunits. Expression of the Torpedo AChR genes in animal cells may alleviate this problem. Intrinsic in this approach is the assumption that the genes coding for the four different subunits are sufficient for the assembly of the receptor-ionophore complex. There are currently no data to support this assumption, although it is a parsimonious one.

\section{ACKNOWLEDGMENTS}

This work was supported by a National Science Foundation predoctoral fellowship (to D.J.A.) and a grant from the Muscular Dystrophy Foundation. We thank Drs. Arthur Karlin and Reid Gilmore for helpful discussions during the course of this work.

\section{REFERENCES}

Anderson, D.J. and G. Blobel. 1981. In vitro synthesis, glycosylation and membrane insertion of the four subunits of Torpedo acetylcholine receptor. Proc. Natl. Acad. Sci. 78: 5598 .

. 1983a. Identification of homo-oligomers as potential intermediates in acetylcholine receptor subunit assembly. Proc. Natl. Acad. Sci. 80: 4359. 
1983b. Immunoprecipitation of proteins from cell-free translations. Methods Enzymol. 96: 111.

1983c. Biosynthesis of acetylcholine receptor in vitro. Methods Enzymol. 96: 367.

Anderson, D.J., P. Walter, and G. Blobel. 1982. Signal recognition protein is required for the integration of acetylcholine receptor $\delta$ subunit, a transmembrane glycoprotein, into the endoplasmic reticulum membrane. J. Cell Biol. 93: 501.

Anderson, D.J., G. Blobel, S. Tzartos, W. Gullick, and J. LINDSTROM. 1983. Transmembrane topology of an early biosynthetic form of acetylcholine receptor $\delta$ subunit studied by limited proteolysis and monoclonal antibodies. J. Neurosci. 3: 1773.

Blobel, G. 1980. Intracellular protein topogenesis. Proc. Natl. Acad. Sci. 77: 1496.

Blobel, G. and B. Dobberstein. 1975. Transfer of proteins across membranes. II. Reconstitution of functional rough microsomes from heterologous components. J. Cell Biol. 67: 852 .

BoeKE, J.D. and P. Model. 1982. Identification of prokaryotic membrane anchor sequence. Proc. Natl. Acad. Sci. 79: 5200.

Braell, W. and H.F. Lodish. 1982. Co-translational insertion of erythrocyte band III into microsomal membranes. Cell 28: 23.

Brockes, J.P. and Z.W. HALl. 1975. Synthesis of acetylcholine receptor by denervated rat diaphragm muscle. Proc. Natl. Acad. Sci. 72: 1368.

Claudio, T. and M. Raftery. 1977. Immunological comparison of acetylcholine receptors and their subunits from species of electric ray. Arch. Biochem. Biophys. 181: 484.

Claudio, T., M. Ballivet, J. Patrick, and S. Heinemann. 1983. Nucleotide and deduced amino acid sequences of Torpedo californica acetylcholine receptor $\gamma$ subunit. Proc. Natl. Acad. Sci. 80: 1111 .

Conti-Tronconi, B.M. and M. Raftery. 1982. The nicotinic cholinergic receptor: Correlation of molecular structure with functional properties. Annu. Rev. Biochem. 51: 491 .

Devillers-Thiery, A., J. Giraudat, M. Bentaboulet, and J.-P. Changeux. 1983. Complete mRNA coding sequence of the acetylcholine binding $\alpha$-subunit of Torpedo marmorata acetylcholine receptor: A model for the transmembrane organization of the polypeptide chain. Proc. Natl. Acad. Sci. 80: 2067.

Dobberstein, B., H. GarofF, G. WARREN, and P.J. RobinsoN. 1979. Cell-free synthesis and membrane insertion of the mouse $\mathrm{H}-2 \mathrm{~d}^{\mathrm{k}}$ major histocompatibility antigen. Cell 17: 759 .

DUNPHY, W.G., E. FrIEs, L.J. URBANI, and J.E. RothMAN 1981. Early and late functions associated with the Golgi apparatus reside in distinct compartments. Proc. Natl. Acad. Sci. 78: 7453 .

Engleman, D.M. and T.A. Steitz. 1981. The spontaneous insertion of proteins into and across membranes: The helical hairpin hypothesis. Cei' 23: 411.

Fambrough, D.M. and P.N. Devreotes. 1978. Newly synthesized acetylcholine receptors are located in the Golgi apparatus. J. Cell Biol. 76: 237.

FroEhNER, S.C. 1981. Identification of exposed and buried determinants of the membrane-bound acetylcholine receptor from Torpedo californica. Biochemistry 20: 4905.

Gilmore, R., P. Walter, and G. Blobel. 1982. Protein translocation across the endoplasmic reticulum. II. Isolation and characterization of the signal recognition particle receptor. J. Cell Biol. 95: 470.

Goldman, B.M. and G. BLobEL. 1981. In vitro biosynthesis, core glycosylation and membrane integration of opsin. $J$. Cell Biol. 90: 236.

Hertzberg, E.L. and N.B. Gilula. 1982. Liver gap junctions and lens fiber junctions: Comparative analysis and calmodulin interaction. Cold Spring Harbor Symp. Quant. Biol. 46: 639.
Hunt, L.A. and D.F. Summers. 1976. Glycosylation of vesicular stomatitis virus glycoprotein in virus-infected HeLa cells. J. Virol. 20: 646 .

Jacobs, S., F.C. Kull, and P. Cuatrecasas. 1983. Monensin blocks the maturation of receptors for insulin and somatomedin C: Identification of receptor precursors. Proc. Natl. Acad. Sci. 80: 1228.

Jessel, T.M., R.E. SiEgel, and G.D. FishbaCh. 1979. Induction of acetylcholine receptors on cultured skeletal muscle by a factor extracted from brain and spinal cord. Proc. Natl. Acad. Sci. 76: 5397.

KaRLIN, A. 1980. Molecular properties of nicotinic acetylcholine receptors. In The cell surface and neuronal function (ed. C.W. Cotman et al.), p. 191. Elsevier/ North-Holland, Amsterdam.

Karlin, A., E. Holtzman, N. Yodh, P. Lobel, J. Wall, and J. HAINFELD. 1983. The arrangement of the subunits of the acetylcholine receptor of Torpedo californica. $J$. Biol. Chem. 258: 6678.

Kistler, J., R.M. Stroud, M.W. Klymkowski, R.A. LAlANCETte, and R.M. FAIRClough. 1982. Structure and function of an acetylcholine receptor. Biophys. $J$. 37: 371 .

Lindstrom, J., B. Einarson, and J.P. Merlie. 1978. Immunization of rats with polypeptide chains from Torpedo acetylcholine receptors causes an immune response to receptors in rat muscle. Proc. Natl. Acad. Sci. 74: 3278.

LiNGAPPA, V.R., F.N. KatZ, H.F. LoDISH, and G. BLOBEL. 1978. A signal sequence for the insertion of a transmembrane glycoprotein. J. Biol. Chem. 253: 8667.

Mendez, B., P. Valenzuela, J.A. Martial, and J.D. BAXTER. 1980. Cell-free synthesis of acetylcholine receptor polypeptides. Science 209: 695.

Merlie, J.P. and R. Sebbane. 1981. Acetylcholine receptor subunits transmit a precursor pool before acquiring $\alpha$ bungarotoxin binding. J. Biol. Chem. 256: 3605 .

Merlie, J.P., J. Hoffler, and R. Sebbane. 1981. Inhibition of glycosylation by tunicamycin blocks the assembly of acetylcholine receptor subunits. J. Biol. Chem. 256: 6995.

Meyer, D.I., E. Krause, and B. Dobberstein. 1982. Secretory protein translocation across membranes-The role of the 'docking protein.' Nature 297: 647.

Milstein, C., G.G. BrownleE, T.M. Harrison, and M.B. Mathews. 1972. A possible precursor of immunoglobulin light chain. Nat. New Biol. 239: 117.

Mostov, K.E., P. DeFoor, S. Fleischer, and G. BLobel. 1981. Co-translation integration of calcium pump protein without signal sequence cleavage. Nature 292: 87.

Noda, M., H. Takahashi, T. Tanaka, M. Toyosato, Y. Furutani, T. Mirose, M. Asai, S. Inayama, T. MiYata, and S. Numa. 1982. Primary structure of the $\alpha$ subunit precursor of Torpedo californica acetylcholine receptor deduced from cDNA sequence. Nature 299: 793.

Noda, M., M. Takahashi, T. Tanabe, M. Toyosato, S. Kikyotani, Y. Furutani, T. Hirose, H. Takashima, S. Inayama, T. MiYata, and S. Numa. 1983. Structural homology of Torpedo californica acetylcholine receptor subunits. Nature 302: 528.

Raftery, M.A., M.W. Hunkapiller, C.B.D. Strader, and L.E. Hood. 1980. Acetylcholine receptor: Complex of homologous subunits. Science 208: 1454.

Robins, P.W., S.C. Hubbard, J. TuRCo, and D.F. WirTh 1977. Proposal for a common oligosaccharide intermediate in the synthesis of membrane glycoproteins. Cell 12: 893 .

Rogers, M.J. and P. Strittmatter. 1975. The insertion of cytochrome $b_{5}$ into natural and artificial lipid bilayer membranes. J. Biol. Chem. 250: 5713.

RothmaN, J.E. and H.F. Lodish. 1977. Synchronised transmembrane insertion and glycosylation of a nascent membrane protein. Nature 269: 775 .

Strader, C.D. and M.A. Raftery. 1980. Topographic studies of Torpedo acetylcholine receptor subunits as a transmembrane complex. Proc. Natl. Acad. Sci. 77: 5807. 
Sumikawa, K., M. Houghton, J.C. Smith, L. Bell, B.M. RichaRDs, and E.A. BARNARD. 1982. The molecular cloning and characterisation of cDNA coding for the $\alpha$ subunit of the acetylcholine receptor. Nucleic Acids Res. 10: 5809 .

Tzartos, S.J. and J.-P. Changeux. 1983. High affinity binding of $\alpha$-bungarotoxin to the purified $\alpha$ subunit and to its 27,000-dalton proteolytic peptide from Torpedo marmorata acetylcholine receptor. Requirement for sodium dodecyl sulphate. EMBO J. 2: 381 .

Walter, P. and G. Blobel. 1980. Purification of a membrane-associated protein complex required for protein translocation across the endoplasmic reticulum. Proc. Natl. Acad. Sci. 77: 7112.

- 1981. Translocation of proteins across the endoplasmic reticulum. III. Signal recognition protein (SRP) causes signal sequence-dependent and site-specific arrest of chain elongation that is released by microsomal membranes. $J$. Cell Biol. 91: 557.

- 1982. Signal recognition particle contains a 7S RNA essential for protein translocation across the endoplasmic reticulum membrane. Nature 299: 691.

Warren, G. and B. Dobberstein. 1978. Protein transfer across microsomal membranes reassembled from separated membrane components. Nature 273: 569.

Wennogle, L.P. and J.-P. Changeux. 1980. Transmembrane orientation of proteins present in acetylcholine receptor-rich membranes from Torpedo marmorata studied by selective proteolysis. Eur. J. Biochem. 106: 381 .

Wennogle, L.P., R. Oswald, T. Saitoh, and J.-P. Changeux. 1981. Dissection of the 66,000 dalton subunit of the acetylcholine receptor. Biochemistry 20: 2492. 